\title{
The Impact of the Hashtag Challenge of the Short-form Video Platform on Brand Recognition and Product Purchase
}

\author{
Dong-Yoon $\mathrm{Kim}^{1}$, Hoon-Sik Yoo ${ }^{2}$ \\ ${ }^{1}$ Student, Department of New Media, Seoul Media Institute of Technology(SMIT), South of Korea, \\ dongyoon0830@gmail.com \\ ${ }^{2}$ Professor, Department of New Media, Seoul Media Institute of Technology(SMIT), South of Korea, \\ hsyoo@smit.ac.kr \\ Corresponding author: Hoon-Sik Yoo
}

\begin{abstract}
This study is aimed to propose an effective advertisement exposure method by analyzing the advertisement attitude of consumers who are exposed to advertisements in the short-form video platform's hashtag challenge. The speed of content upgrades is gradually accelerating, and people's media consumption habits are also changing along with the change of the content usage environment like the advancement of technologies (e.g., smart devices) and the universalization of the Internet. With people moving from a text- and image-oriented media platform to a video-oriented platform, video has become a popular form of media consumption in the current mobile era. Short-form video platforms have emerged as a new advertisement marketing outlet as short videos surpass long videos in influencing the public. Recognizing a lack of research on TikTok advertisement, this study examined the attitude of consumers who viewed advertisements in the TikTok hashtag challenge by defining TikTok as a representative short-form video platform and using a survey to collect data. To this end, a survey was conducted to 252 consumers who have watched TikTok advertisements to examine their acceptance attitude. The research tool for each item in the questionnaire selected whether to watch the hashtag challenge, participate in the hashtag challenge, recognize the hashtag challenge brand, and purchase the hashtag challenge brand product. The study found a certain degree of advertising effect on product purchase, although the effect size was small. Drawing on the findings, the study suggested a guideline for advertisement exposure suitable for one-person media creators and marketing practitioners who want to use short-form video platforms for advertising.
\end{abstract}

Keywords: Short-form Video Platform, Short-form Content, Hashtag Challenge, Influencer, Advertisement Marketing, Advertisement Acceptance

\section{Introduction}

People have started to prefer short and interesting information as the mobile Internet is spreading and people's life rhythms are getting faster. Short-form content platform users have been increasing every year, and more people have begun to pay attention to short-form content[1]. Furthermore, the recent marketing trend is penetrating through the $\mathrm{MZ}$ generations (millennials and $\mathrm{Z}$ generations). The $\mathrm{Z}$ generation, born in the 1990s through 2000s, yearns for new content, and people who want to create their own business as creators have a considerable interest in the next-generation media platform. It is the short-form content platform known as TikTok where this interest and direction are converged.

Received: April 05, 2021; $1^{\text {st }}$ Review Result: May 24, 2021; $2^{\text {nd }}$ Review Result: July 12, 2021

Accepted: August 30, 2021 
In early 2020, when the number of people living indoors increased due to the COVID-19 pandemic, the cumulative number of app downloads reached two billion (as of May 2020), and the number of Korean users of TikTok exceeded three million. Due to the change in modern people's leisure life, people have started to look for short-form content like TikTok, which is easy to use and produce in a new media environment[1]. Short-form content platforms such as TikTok, Lil's, and YouTube Shorts are becoming more valuable; they provide various attractions by serving as a communication medium and an information/content collection source in a short video of 15 to 30 seconds. Short-form content platforms are being used as a playground where people communicate with other people and create a new culture through producing and consuming content. Considering that the $\mathrm{Z}$ generation is expected to emerge as a core layer of consumption three to five years later, companies using short-form content platforms as their marketing outlet are on the rise. Due to the mighty diffusion power of mobile-oriented video shooting and production, the hashtag challenge, a representative function of the short-form content platform, is creating an international ripple effect by inducing an explosive number of people to participate.

Observing this favorable condition, TikTok is attempting to expand its marketplace through marketing conferences. The creator marketplace for recruiting TikTok creators for hashtag challenge, brand effect, and in-feed advertisement is a representative example, and the impact this move will bring to the advertising market in the future is drawing attention. This study conducted an online survey on TikTok, which is emerging as a powerhouse of short-form content platforms, to examine the changes in users' advertisement attitude and product purchase behavior, focusing on advertisements in the TikTok hashtag challenge. Furthermore, the researchers investigated the impact of consumers' viewing of advertisements in TikTok on their brand recognition and product purchase and suggested a way to expose advertisements to TikTok users more effectively. To this end, the hypothesis of this study is as follows.

Compared to existing TikTok advertisements, TikTok hashtag challenge ads have positive impact on consumers' acceptance attitude (whether they participate in the hashtag challenge, recognize the hashtag challenge brand, and experience purchasing hashtag challenge brand products).

\section{Related Research}

\subsection{Definition of Short-form Content and TikTok}

A move from a text- and image-oriented media platform to a video-oriented platform has made video a new media consumption trend in the current mobile era[2]. An analysis of mobile device data reveals that short video has surpassed long video as a major part of content consumption[3].

Short-form content (a.k.a., short video or short clip video) containing short-length funny videos that feature personal daily life or dancing is edited using various functions and shared with people. It can be easily created by inserting background music and various special effects, even without a specialized video editing program[4]. It is categorized in a wide variety of genres, such as descriptions of product functions, comics, fashion trends, social issues, interviews, public advertisements, idea introductions, and business advertisements[3].

The short-form platform that shares short-form content has explosively grown first in China in 2016. According to an analytic report on China's short-form content platforms (Qimai, 2018), a representative short-form content platform is Douyin, the predecessor of TikTok, while various short-form content platforms like Kuaishou and Miao Pai exist in the Chinese market[5]. Douyin is the Chinese version of TikTok, of which main content is UGC(user generated contents) content with music; $85 \%$ of its current users are under the age of 24 , and it is the fastest-growing short-form content platform in China. Based 
on its success in the Chinese market, Douyin launched a service in the U.S. and Korean markets in 2017 under the name of TikTok and has developed into the most popular social platform that provides services in more than 150 global markets, including the U.S., over the recent two to three years[6].

\subsection{Short-form Platform TikTok and Advertising}

TikTok is a global video platform that can create and share short-form (15- to 60-second-long) videos. Since exceeding 1.5 billion downloads in 2019, TikTok has emerged as the center of the short-form content platform. Short-length videos require relatively little time and labor, which means that creators are less burdened with producing and participating in their own content. Therefore, TikTok functions as a play culture for the $\mathrm{MZ}$ generations who quickly produce and consume content and pay attention to new things. With the popularity of the TikTok platform that provides short-form content and the growth of the corresponding short-form content market, many companies are interested in the consumption pattern of generations who view short-form content. Utilizing short-form content in marketing, various advertising strategies are being implemented to target the $\mathrm{MZ}$ generations who prefer short-form content and to imprint the brand identity on them within a short time[7].

\subsection{Features of Hashtag Challenge Ads}

TikTok's hashtag challenge ads are perceived to have higher interaction with consumers compared to other types of advertisements in TikTok. Hashtag Challenge ads are a type of advertisement that allows all users to participate in advertisements, not unilaterally advertising by advertisers, which reduces the distance between users and advertisements and naturally reduces the reluctance to advertise. Additionally, interactive hashtag challenge advertising content drives consumers to like and participate. Since many of the hashtag challenge advertisements are UGC (User-Generated Contents) content filmed by ordinary people, message recipients form intimacy and trust in product introduction and recommendation compared to other advertisements[8]. The familiarity with advertising brings empathy, leads to curiosity and affinity for the products in the advertisement, and leads to participation in advertising activities such as sharing or opening a link to purchase. The easier it is to use hashtag challenge advertisements, the more likely consumers are to immerse themselves in and participate in advertisements[9].

\subsection{TikTok Content Type and Advertisement Challenge Case}

Lee (2020) classified the types of short-form video content based on communication type (discourse vs. dialogue) and the content creation method (top-down vs. bottom-up). According to the classification, the short-form content in TikTok is divided into two types: dialogue-top-down type and dialoguebottom-up type[10].

Participatory campaigns or branding contents belong to the dialogue-top-down type, which is designed as a marketing strategy to bypass the unconsciousness of viewers who do not want to be exposed to advertisements by mingling advertisement into content for the delivery and spread of messages. This is a media that creates a 'movie-like advertisement' and 'drama-like advertisement' in marketing and branding, paying attention to the concept of storytelling and having consumers who view the advertisement participate in creating and sharing content through a dialogue-type communication. Representative examples of the dialogue-top-down type include the Random Song Challenge, a participatory branding video created for album marketing by singer Zico, and the CA Swag Challenge, a participatory branding video using augmented reality stickers for the game character of Crazy Arcade [10]. 

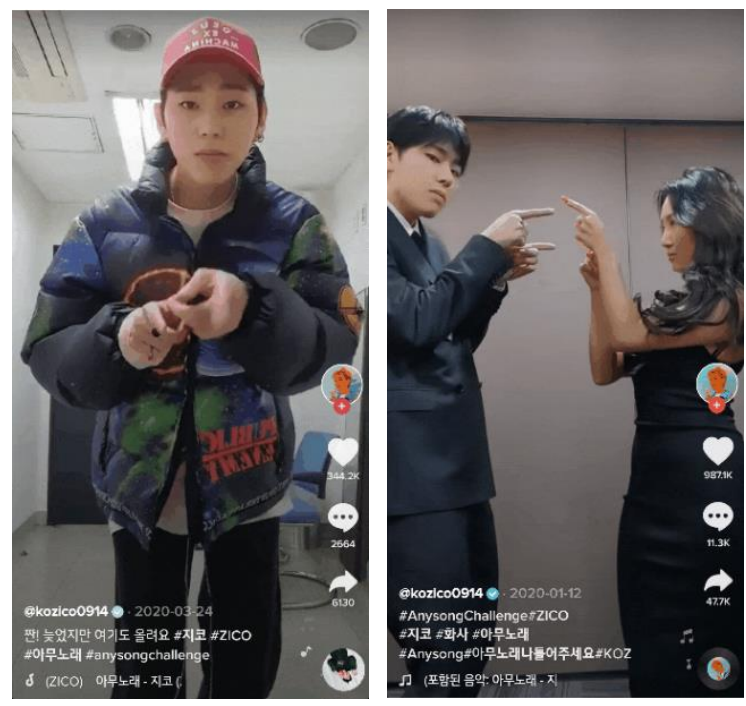

[Fig. 1] TikTok Random Song Challenge

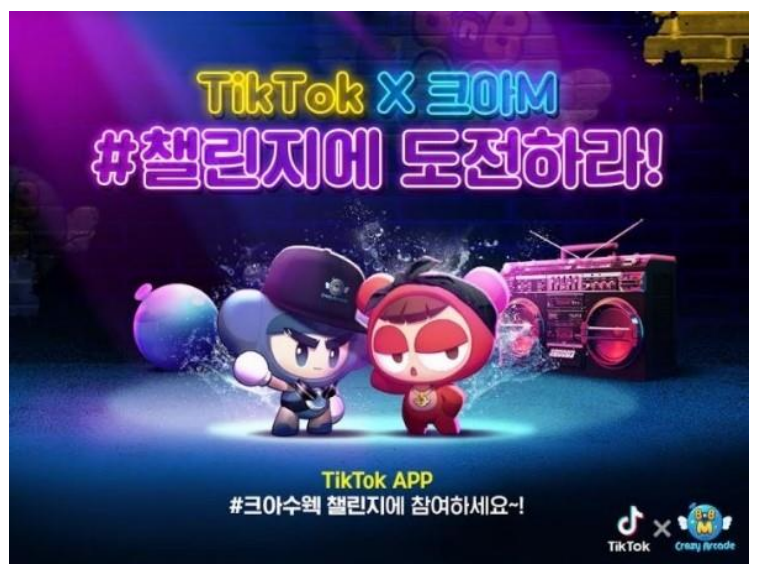

[Fig. 2] TikTok CA Swag Challenge

Dialogue-bottom-up type content appears in the form of videos in which users create and share messages they want. TikTok provides editing tools optimized for music, background screens, special effects, and montage techniques; entertainment content, including music, comedy, and situational drama, is popularly created in TikTok. The characteristics of TikTok contents are well revealed through the hashtag challenge function and the duet function that combines the images of other users and their own. An influencer is created in the process of sharing and spreading content, and a video containing a person's daily life combined with branding and commerce appears as a means of marketing for individual businesses or companies based on the inner intimacy formed between the influencer and their followers[10].

\section{Research Methods}

\subsection{Scope}

Previous studies on short-form platforms have focused on TikTok users' motives and behaviors. However, research participants were mainly limited to Chinese, and few studies were conducted on advertising despite the increasing number of users moving from long-form platforms to short-form 
platforms and the various types of advertisement (e.g., brand take-over, top-view, in-feed, and branded hashtag challenge) in TikTok. To fill this research gap, the study analyzed the attitude of consumers who viewed advertisements in through the hashtag challenge, which is the most representative form of TikTok advertisements. This study examined users' experience viewing the TikTok hashtag challenge, participating in the TikTok hashtag challenge, recognizing a brand in the TikTok hashtag challenge, and purchasing branded products in the TikTok hashtag challenge.

\subsection{Survey}

A survey method was employed to collect data from the general public who viewed the TikTok shortform platform. This survey was designed to identify the characteristics of consumers' ad acceptance attitude to advertisements in short-form content (hashtag challenge) in TikTok. Respondents to this study were limited to consumers who have watched TikTok advertisements. A total of 252 people responded to the survey through a Google Forms for a week from January 7 to 14, 2021. The demographic information of the survey participants is presented in [Table 1]. The survey questionnaire was designed to collect data on respondents' experience viewing the hashtag challenge, participating in the hashtag challenge, recognizing a brand in the hashtag challenge, and purchasing branded products in the hashtag challenge.

[Table 1] Demographic Information of the Participants

\begin{tabular}{|c|c|c|}
\hline \multirow{2}{*}{ Total respondents } & Frequency \\
\hline \multirow{3}{*}{ Sex } & Male & 252 \\
\cline { 2 - 3 } & Female & 81 \\
\hline \multirow{3}{*}{ Age } & $20 \mathrm{~s}$ & 171 \\
\cline { 2 - 3 } & $30 \mathrm{~s}$ & 129 \\
\cline { 2 - 3 } & $40 \mathrm{~s}$ & 104 \\
\cline { 2 - 3 } & $50 \mathrm{~s}$ & 15 \\
\hline
\end{tabular}

\section{Findings}

\subsection{Viewing of TikTok Hashtag Challenge}

A majority of the respondents $(\mathrm{n}=252)$ viewed the TikTok hashtag challenge $(79.4 \%$, ) with only $20.6 \%$ having no experience viewing the hashtag challenge, as presented in [Table 2].

[Table 2] Viewing of TikTok Hashtag Challenge

\begin{tabular}{|c|c|c|c|c|}
\hline & & Frequency & Viewed (\%) & Not viewed (\%) \\
\hline \multicolumn{2}{|c|}{ Total respondents } & 252 & $200(79.4)$ & $52(20.6)$ \\
\hline \multirow{2}{*}{ Sex } & Male & 81 & $55(67.9)$ & $26(32.1)$ \\
\cline { 2 - 5 } & Female & 171 & $145(84.8)$ & $26(15.2)$ \\
\hline \multirow{2}{*}{ Age } & $20 \mathrm{~s}$ & 129 & $106(82.2)$ & $23(17.8)$ \\
\hline
\end{tabular}




\begin{tabular}{|c|c|c|c|c|}
\hline & $30 \mathrm{~s}$ & 104 & $85(81.7)$ & $19(18.3)$ \\
\cline { 2 - 5 } & $40 \mathrm{~s}$ & 15 & $5(33.3)$ & $10(66.7)$ \\
\cline { 2 - 5 } & $50 \mathrm{~s}$ & 4 & $4(100.0)$ & $0(0.0)$ \\
\hline
\end{tabular}

\section{[Viewing of TikTok Hashtag Challenge]}

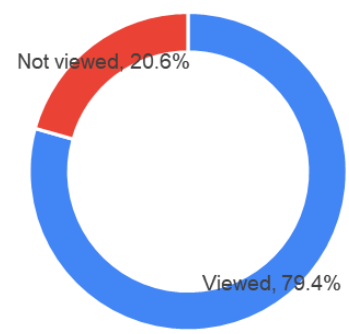

- Viewed - Not viewed

[Fig. 3] Viewing of TikTok Hashtag Challenge

\subsection{Participation in TikTok Hashtag Challenge}

About $40.1 \%$ of the respondents turned out to have experienced participating in the hashtag challenge, while the remaining $59.9 \%$ have not participated prior this research.

\section{[Participation in TikTok Hashtag Challenge]}

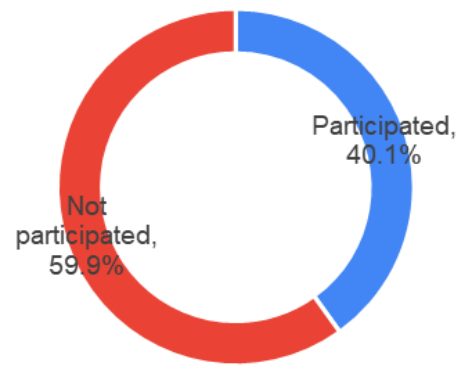

- Participated - Not participated

[Fig. 4] Participation in TikTok Hashtag Challenge

[Table 3] Participation in TikTok Hashtag Challenge

\begin{tabular}{|c|c|c|c|c|}
\hline & & Frequency & Participated (\%) & Not participated (\%) \\
\hline \multicolumn{2}{|c|}{ Total respondents } & 252 & $101(40.1)$ & $151(59.9)$ \\
\hline Sex & Male & 81 & $27(33.3)$ & $54(66.7)$ \\
\hline
\end{tabular}




\begin{tabular}{|c|c|c|c|c|}
\hline & Female & 171 & $74(43.3)$ & $97(56.7)$ \\
\hline \multirow{4}{*}{ Age } & $20 \mathrm{~s}$ & 129 & $51(39.5)$ & $78(60.5)$ \\
\cline { 2 - 5 } & $30 \mathrm{~s}$ & 104 & $45(43.3)$ & $59(56.7)$ \\
\cline { 2 - 5 } & $40 \mathrm{~s}$ & 15 & $3(20.0)$ & $12(80.0)$ \\
\cline { 2 - 5 } & $50 \mathrm{~s}$ & 4 & $2(50.0)$ & $2(50.0)$ \\
\hline
\end{tabular}

\subsection{Brand Recognition in TikTok Hashtag Challenge}

Regarding brand recognition in the TikTok hashtag challenge, a majority of the respondents turned out to be positive $(50.8 \%)$ or very positive $(15.9 \%)$, while only about $4 \%$ were negative. The percentage of the respondents with a neutral position was $29.4 \%$.

\section{[Brand Recognition in TikTok Hashtag Challenge]}

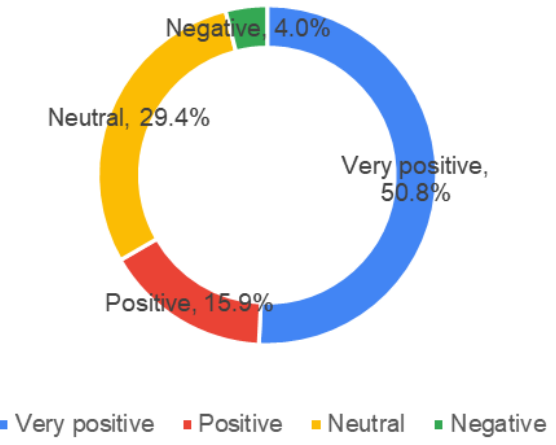

[Fig. 5] Brand Recognition in TikTok Hashtag Challenge

[Table 4] Brand Recognition in TikTok Hashtag Challenge

\begin{tabular}{|c|c|c|c|c|c|c|}
\hline & Frequency & Negative (\%) & Neutral (\%) & Positive (\%) & $\begin{array}{c}\text { Very positive } \\
(\%)\end{array}$ \\
\hline \multirow{2}{*}{ Total respondents } & 252 & $10(4.0)$ & $74(29.4)$ & $128(50.8)$ & $40(15.9)$ \\
\hline \multirow{3}{*}{ Sex } & Male & 81 & $4(4.9)$ & $22(27.2)$ & $42(51.9)$ & $13(16.0)$ \\
\cline { 2 - 7 } & Female & 171 & $6(3.5)$ & $52(30.4)$ & $86(50.3)$ & $27(15.8)$ \\
\hline \multirow{3}{*}{ Age } & $20 \mathrm{~s}$ & 129 & $3(2.3)$ & $37(28.7)$ & $67(51.9)$ & $22(17.1)$ \\
\cline { 2 - 7 } & $30 \mathrm{~s}$ & 104 & $7(6.7)$ & $28(26.9)$ & $55(52.9)$ & $14(13.5)$ \\
\cline { 2 - 7 } & $40 \mathrm{~s}$ & 15 & $0(0.0)$ & $8(53.3)$ & $6(40.0)$ & $1(6.7)$ \\
\hline
\end{tabular}

\subsection{Experience in Purchasing Branded Uppercase products in TikTok Hashtag Challenge}

Finally, the results show that the TikTok hashtag challenge converted more than a quarter of the 
respondents into purchasers of the products advertised in the said challenge. About $32.5 \%$ of the respondents reported having an experience purchasing branded products in the TikTok hashtag challenge, with the remaining $67.5 \%$ reporting no experience of buying branded products in the said challenge.

[Experience in Purchasing Branded products in TikTok Hashtag Challenge]

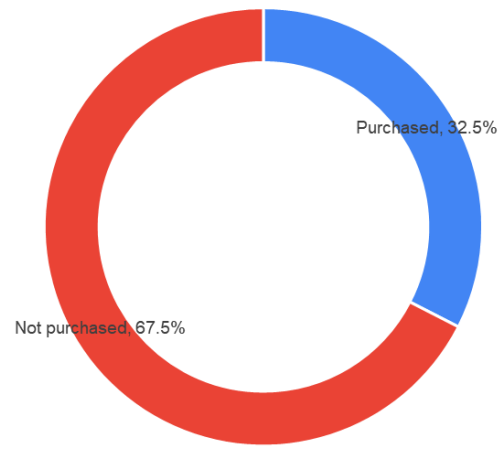

- Purchased - Not purchased

[Fig. 6] Experience in Purchasing Branded Uppercase products in TikTok Hashtag Challenge

[Table 5] Experience in Purchasing Branded Uppercase products in TikTok Hashtag Challenge

\begin{tabular}{|c|c|c|c|c|}
\hline & & Frequency & Purchased (\%) & Not purchased (\%) \\
\hline \multirow{2}{*}{ Total respondents } & 252 & $82(32.5)$ & $170(67.5)$ \\
\hline \multirow{3}{*}{ Sex } & Male & 81 & $23(24.8)$ & $58(71.6)$ \\
\cline { 2 - 5 } & Female & 171 & $59(34.5)$ & $112(65.5)$ \\
\cline { 2 - 5 } & $20 \mathrm{~s}$ & 129 & $37(28.7)$ & $92(71.3)$ \\
\cline { 2 - 5 } & $30 \mathrm{~s}$ & 104 & $36(34.6)$ & $68(65.4)$ \\
\cline { 2 - 5 } & $40 \mathrm{~s}$ & 15 & $7(46.7)$ & $8(53.3)$ \\
\cline { 2 - 5 } & $50 \mathrm{~s}$ & 4 & $2(50.0)$ & $2(50.0)$ \\
\hline
\end{tabular}

\section{Conclusion}

This study was conducted to provide primary data to verify the effectiveness of consumer acceptance attitudes of hashtag challenge ads by identifying whether consumers who have watched TikTok ads participated in hashtag challenge, hashtag challenge brand recognition, and hashtag challenge brand purchasing experience.

For this study, a survey was conducted on 252 consumers who have previously watched TikTok advertisements, hypothesizing that TikTok hashtag challenge advertisement will positively impact consumers' acceptance attitude (hashtag challenge brand recognition, hashtag challenge brand purchase experience). The analysis confirmed that the research model proposed to explain the relationship between TikTok hashtag challenge advertising variables was appropriate for explaining these 
relationships integrally. The effectiveness between the influence and the outcome factors was significant.

The main results of this study are as follows. First, more than half of TikTok users watched hashtag challenge ads and are active in participating in themselves. Most TikTok users have watched the hashtag challenge (79.4\%), and the remaining $20.6 \%$ have never watched the hashtag challenge. In addition, $40.1 \%$ of TikTok users have participated in the hashtag challenge, and $59.9 \%$ have not participated in the hashtag challenge. Second, the hashtag challenge advertisement felt by TikTok users appears to have more positive views than average. In terms of brand recognition conducted in the hashtag challenge, 50.8 percent received the most responses, followed by moderate ( 29.4 percent) and very positive (15.9 percent). Third, it is meaningful that the TikTok hashtag challenge causes the purchase conversion of the brand product of the advertisement in more than a quarter of people. $32.5 \%$ of TikTok users have purchased brand products from hashtag challenge, while the remaining $67.5 \%$ have never purchased brand products from hashtag challenge. Thus, most consumers who have watched hashtag challenge ads can consider using these ads to promote brand recognition and product sales among TikTok ads, given that they lead to positive perceptions and actual purchase conversions.

The significance and implications of this study are as follows. First, while previous studies on the short-form content platform relied on Chinese people who used Douyin, the predecessor of TikTok, this study extended the research participant to Korean people who had an experience with TikTok, the global version of Douyin. Second, while previous studies on TikTok were focused on the types of short-form video content or the motivation of viewers using the platform, this study expanded the scope of research to investigate TikTok users' acceptance of advertisements in the TikTok hashtag challenge.

The limitations and suggestions of this study for future research are as follows. First, the subjects of this study are unevenly distributed across gender and age groups, resulting in a limitation in generalizing the findings. Additional research needs to be conducted with a sample that better represents the population in terms of gender and age. Second, the scale to measure brand recognition in the hashtag challenge needs to be extended to a five-point Likert scale, including "very negative," to make the scale balanced between positive and negative to capture more accurate responses. Third, future research needs to add other short-form platforms such as YouTube Short, Instagram Reels, Naver Moment, and Snapchat that appeared after TikTok to investigate the effect of the hashtag challenge on brand recognition and product purchase. Finally, future research needs to expand the age range of survey respondents to teenagers who represent the $\mathrm{Z}$ generation.

Among one-person media channels, short-form content platforms are a newly emerging field. As advertisement marketing through short-form content platforms is increasing, research on consumers' acceptance of the advertisement through short-form content platforms is expected to provide TikTok creators and companies with directions for more effective content creation and advertisement exposure.

\section{References}

[1] W. Jung, D. Choi, A Study on the Relationship between the Short Clip Consumption of Contemporary People's Leisure Time - Focused on "TikTok," Proceedings of the Korea Contents Association Conference, (2019), May 17 18, Sangmyung University, Seoul, Korea, pp.363-364.

[2] H. Kim, H. Pyo, M. Han, Analysis on the Influences and Effects of eWOM (electronic Word of Mouth) of Power Blog Post as a Source of Advertisement, Korean Journal of Advertising and Public Relations, (2011), Vol.13, No.4, pp.315362.

[3] Y. Ryu, A Study on the Factors Affecting Continuous Usage Intention of Short Video Apps: Focus on Chinese TIK TOK(DOU YIN)APP, Korea Speech, Media \& Communication Association Conference, (2019), Jun 15; Seoul, Korea. 
[4] Social Media Behavior and Advertising Contact Attitudes Analysis Report, DMC Media, (2020), https://www.dmcreport.co.kr/report/surveyReport/freeView?reportcode=DMCSRF20200047\&drtopdeth=RPT_TYP E_3\&keyword_type=REPORT_KEYWORD_16.

[5] S. Jinhua, O. Izuchukwu, K. Seo, D. Jung, A Study on the Motivation and Behavior of Short Video(Tiktok): Focused on Korean and Chinese teenagers, North-East Asian Cultures' International Conference, (2020), October 31; Busan, Korea.

[6] Y. Jung, H. Wi, Effects of Perceived Quality of TikTok on User Satisfaction and Continuance Intention: and the Role of Commercial Viability, The Journal of the Korean Contents Association, (2020), Vol.20, No.6, pp.77-89.

[7] H. Kim, S. Oh, S. Cho, Short-form Content Trend, Marketing, (2020), Vol.54, No.7, pp. 60-69.

[8] Ruiyao Ma, Sojung Kim, Use, Motivations, and Responses of TikTok as an Advertising Channel, The Journal of the Korea Contents Association, (2021), Vol.21, No.2, pp.507-519.

[9] Ruiyao Ma, Sojung Kim, Factors Affecting Advertising Effectiveness in TikTok: Focusing on Advertising Engagement, Journal of Practical Research in Advertising and Public Relations, (2021), Vol.14, No.1, pp.7-32.

[10] J. Lee, A Study on Types of Short-form Video Contents, Humanities Contents, (2020), No.58, pp.121-139. 